\title{
Multiple Forms of Human Plasma Renin Substrate
}

\author{
Peter Eggena, Hiroshi Hidaka, Jack D. Barrett, and Mohinder P. Sambhi, \\ Hypertension Division of the Veterans Administration Hospital, Sepulveda, \\ California 91343 and the Department of Medicine, University of California \\ at Los Angeles, Los Angeles, California 90024
}

A B S T R A C T The objective of this investigation was to determine whether heterogeneity of plasma renin substrate could be observed in states of steroid excess and various forms of hypertensive disease. In states of stimulated renin substrate production by estrogens or glucocorticoids, multiple forms of renin substrate were apparent when stimulation was excessive. Stimulation of substrate production caused by uremia associated with hypertension showed similar results. None, or only trace quantities of the additional forms of renin substrate were evident in subjects with normal or suppressed levels of plasma renin substrate. The additional forms of renin substrate could be distinguished from the normal form on the basis of crossreactivity with a specific antiserum to the normal form, electrophoretic mobility, and kinetic rate constants. Differences in rate constants of the various forms of plasma renin substrate may account for the altered rate of the renin reaction associated with several states of hypertension. In plasma of patients with renovascular hypertension, significant quantities of a protein which cross-reacted with the antiserum but could not generate angiotensin I were observed.

\section{INTRODUCTION}

We have previously described the purification of renin substrate from normal human plasma (1) and the development of a direct radioimmunoassay for this protein (2). The direct assay for renin substrate in normal human plasma was validated by demonstrating a 1:1 correlation to substrate measured by the generation of angiotensin I by prolonged incubation with exogenous homologous renin (indirect method). Results of preliminary experiments indicated, however, that in plasma samples in which renin substrate concentration was elevated at least $2 \mathrm{SD}$ above normal as measured by angiotensin I generation, the direct as-

Dr. Hidaka's present address is the University of Tokyo, First Department of Medicine, Tokyo, Japan.

Received for publication 14 November 1977 and in revised form 27 March 1978. say underestimated the renin substrate concentration suggesting that other forms of renin substrate were present in these plasmas which could be cleaved by renin to generate angiotensin I, but did not crossreact with the antiserum produced against the renin substrate of normal plasma (2). The following study examines the various forms of plasma renin substrate in several clinical states associated with hypertension and in patients receiving agents known to stimulate renin substrate synthesis.

\section{METHODS}

Blood was collected in $15 \mathrm{mM}$ EDTA and plasma samples were stored at $-20^{\circ} \mathrm{C}$. Plasma renin substrate was quantitated by two methods: (a) direct, a detailed description of this method has previously been reported by Eggena et al. (2). Briefly, ${ }^{125}$ I-labeled renin substrate and its specific antiserum (produced in rabbit) were added to a diluted plasma sample and equilibrated at $4^{\circ} \mathrm{C}$ for $18 \mathrm{~h}$. The bound and free antigens are separated after a second equilibration with goat anti-rabbit gamma globulin by centrifugation. (b) Indirect, plasma was incubated for $3 \mathrm{~h}$ at $37^{\circ} \mathrm{C}$ in the presence of angiotensinase inhibitors (3) with excess homologous renal renin (4) to convert all renin substrate to angiotensin I (5). Generated angiotensin was quantitated by radioimmunoassay (5). Plasma renin activity (PRA) ${ }^{1}$ was determined by the method of Barrett et al. (3). Polyacrylamide gel electrophoresis at $\mathrm{pH} 4.3$ and 8.4 were performed in $7 \%$ polyacrylamide (6). Isoelectric focusing in $7 \%$ polyacrylamide was performed according to the method of Lim and Tadayyou (7). Proteins were extracted from $2-\mathrm{mm}$ gel slices in 0.05 Tris- $\mathrm{HCl}, \mathrm{pH} 7.4$ with a Gilson Aquagel extractor (Gilson Medical Electronics, Inc., Middleton, Wis.).

\section{RESULTS}

Normal and essential hypertensive subjects. Results of the direct and indirect measurement of renin substrate in plasma samples from normotensive subjects and patients with essential hypertension are shown in Fig. 1. The data indicate a good correlation between the results of the two assay techniques $(y$ $=1.12 x+4, r=0.78, P<0.01, n=28$ ); no statistically

${ }^{1}$ Abbreviations used in this paper: PRA, plasma renin activity; Rf, running front ratio. 


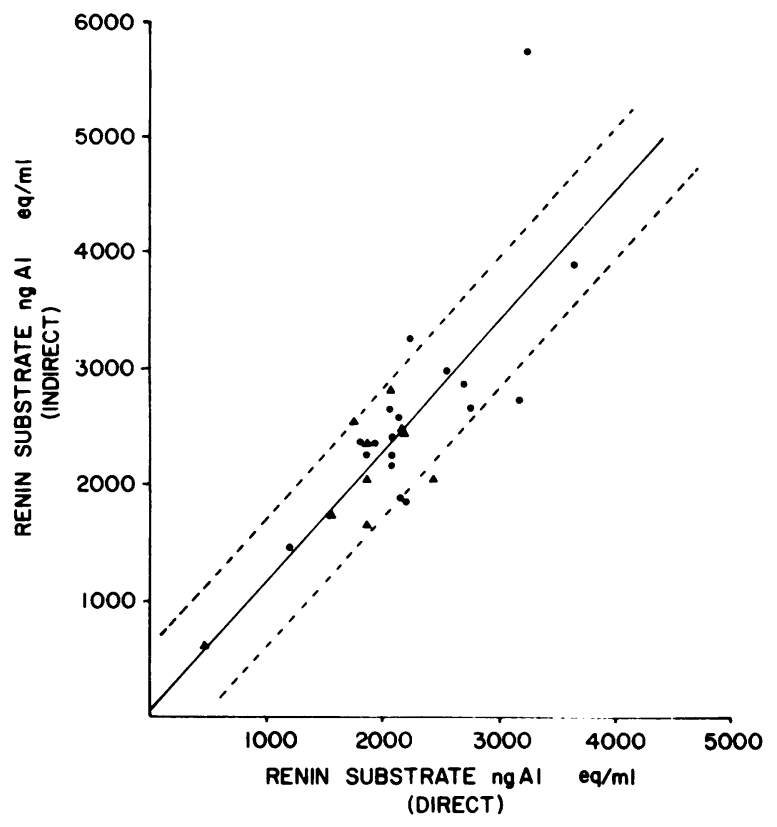

FIGURE 1 Correlation between direct and indirect assay for plasma renin substrate in normal (O) and essential hypertensive subjects $(\boldsymbol{\Delta})$. The solid line indicates the linear least square regression line, the dashed line indicates $1 \mathrm{SD}$. Direct and indirect indicate method of renin substrate assay.

significant difference was evident between plasma from normotensive and hypertensive subjects. On isoelectric focusing in polyacrylamide gel (pH 4-6), all plasma samples showed a broad substrate peak with an isoelectric point at $\mathrm{pH}$ 4.7. Results of polyacrylamide gel electrophoresis at $\mathrm{pH} 8.4$ and subsequent quantitation of substrate by both assay methods are shown in Fig. 2A. One major peak of substrate giving equivalent substrate concentrations by both assay methods is observed. The running front ratio (Rf) of this substrate fraction on polyacrylamide gel electrophoresis was $0.65 \pm 0.05$ (SD). In several subjects, trace quantities of substrate could be detected at $\mathbf{R f}=\mathbf{0 . 3 5}$ \pm 0.05 (SD) and $0.16 \pm 0.02(\mathrm{SD})$ by the angiotensingeneration method (Fig. 2A). Direct radioimmunoassay, although of sufficient sensitivity, did not detect these two minor components, indicating a lack of crossreactivity of these forms of renin substrate with the substrate antibody. Electrophoresis of normal plasma at pH 4.3 demonstrated one major peak at the origin of the gel and a trace of a second peak which reacted with the substrate antiserum (Fig. 2C), but did not generate angiotensin I when incubated with renin. The concentration of this minor component increased upon preincubation of plasma with added homologous renin (Fig. 2D). The increase in plasma concentration of this component determined after polyacrylamide gel electrophoresis (pH 4.3) was always accompanied by a simultaneous decrease in renin substrate concentration and an increase in angiotensin $I$.

Oral contraceptives, pregnancy, and steroids. Measurement of renin substrate in plasma of female subjects taking oral contraceptives confirmed previous studies reporting elevated plasma renin substrate concentrations, as measured by angiotensin I generation $(8,9)$. When the plasma renin substrate levels of these women were below $\cong 6,000 \mathrm{ng}$ angiotensin $\mathrm{I} \mathrm{eq} / \mathrm{ml}$, an excellent linear correlation between both assay methods was obtained (open circles in Fig. 3, $y=1.08$ $+450, r=0.96, n=7$ ). However, when substrate concentrations were above this level, closed circles in Fig. 3 , a discrepancy between the two assay methods was evident; the indirect method giving significantly higher plasma substrate concentrations than the direct assay $(9,600 \pm 3,020$ [SD] vs. $3,100 \pm 1,260 \mathrm{ng}$ angiotensin $\mathrm{I} / \mathrm{ml}$ [SD], $P<0.01)$. The polyacrylamide gel electrophoresis patterns ( $\mathrm{pH}$ 8.4) of these plasma samples with high substrate concentrations (Fig. 2B), demonstrated not only a high substrate level at $\mathrm{Rf}$ $=0.65$ corresponding to the substrate of normal plasma, but also an increase in the renin substrate forms with $\mathrm{Rf}=0.16$ and 0.35 , which were occasionally seen as trace quantities in normal plasma as measured by angiotensin I generation. These additional substrate forms did not cross-react with the substrate antiserum. Similarly, multiple forms of substrate were also observed in two of five women during the third trimester of pregnancy. Trace quantities of the multiple forms may have gone undetected in the three pregnant subjects showing a 1:1 correspondence. Postpartum, the plasma of the two women with multiple forms, contained only the normal form of renin substrate and a 1:1 correspondence between both assay methods was again evident. Multiple forms of substrate were also found in one of three patients on glucocorticoid therapy. Despite the multiple forms of substrate observed on polyacrylamide gel electrophoresis, a single broad peak of substrate with an isoelectric point of 4.7 was found after isoelectrofocusing in all plasmas by either substrate assay.

A patient with Cushing's syndrome giving a higher substrate concentration when measured by angiotensin I generation, as compared to direct assay, showed only the normal form of renin substrate upon polyacrylamide gel electrophoresis at $\mathrm{pH} 4.3$ and 8.4. However, upon isoelectric focusing of this plasma sample, an additional renin substrate peak with an isoelectric point at $\mathrm{pH} 4.3$ was observed. This substrate fraction was capable of generating angiotensin $\mathrm{I}$, but did not cross-react with the antiserum to normal human renin substrate (Fig. 4).

Uremia, advanced essential, malignant, and renovascular hypertension. Renin substrate determinations by both methods were performed using plasma 

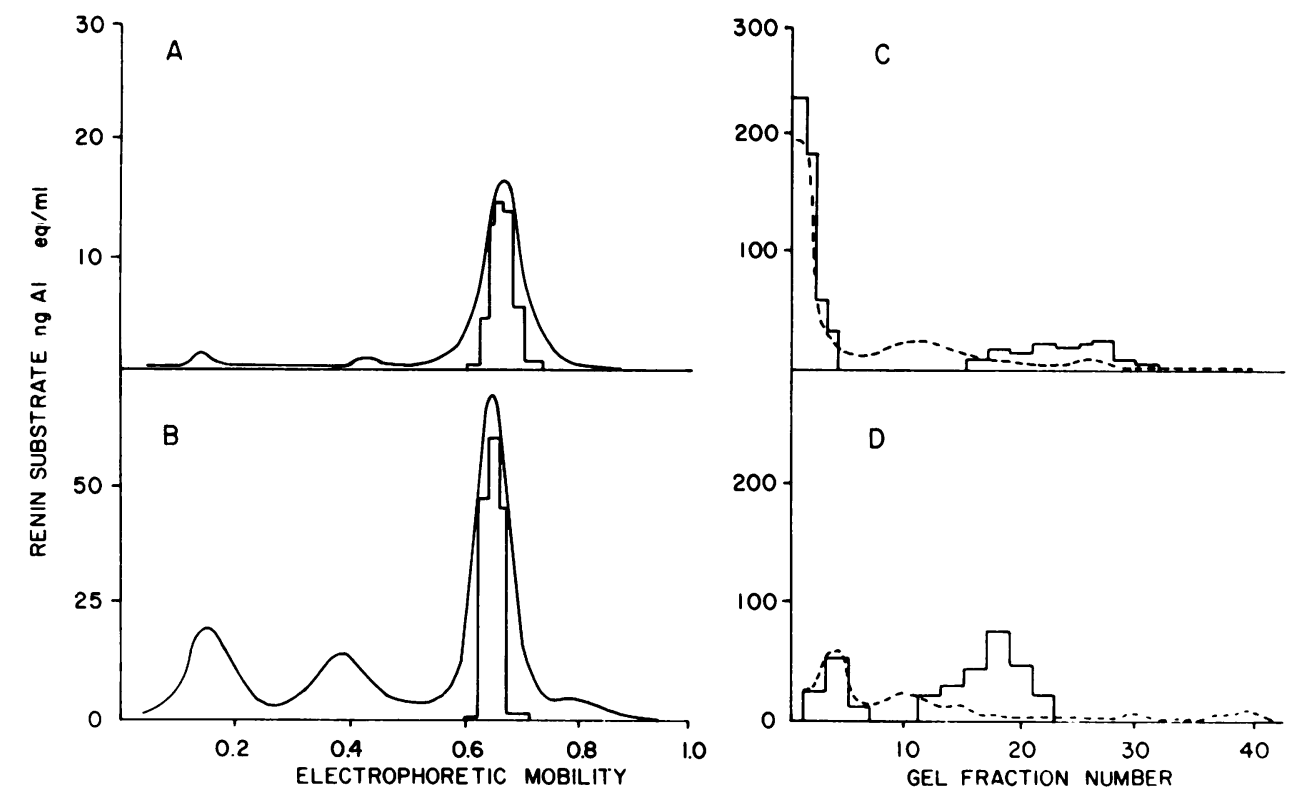

FIGURE 2 Polyacrylamide gel electrophoresis of plasma. (A) normal human plasma, electrophoresis at pH 8.4; bar graph indicates renin substrate determined by direct and continuous line by indirect radioimmunoassay. (B) plasma of women on oral contraceptives with multiple forms of renin substrate, electrophoresis at $\mathrm{pH} 8.4$; bars and line as indicated in (A). (C) normal human plasma, electrophoresis at $\mathrm{pH} 4.3$; bars indicate direct, dashed lines indirect radioimmunoassay. (D) electrophoresis at pH 4.3 of normal human plasma after incubation at $37^{\circ} \mathrm{C}$ for $1 \mathrm{~h}$ with exogenous homologous renal renin; bars at dashed line as described in $(\mathrm{C})$.

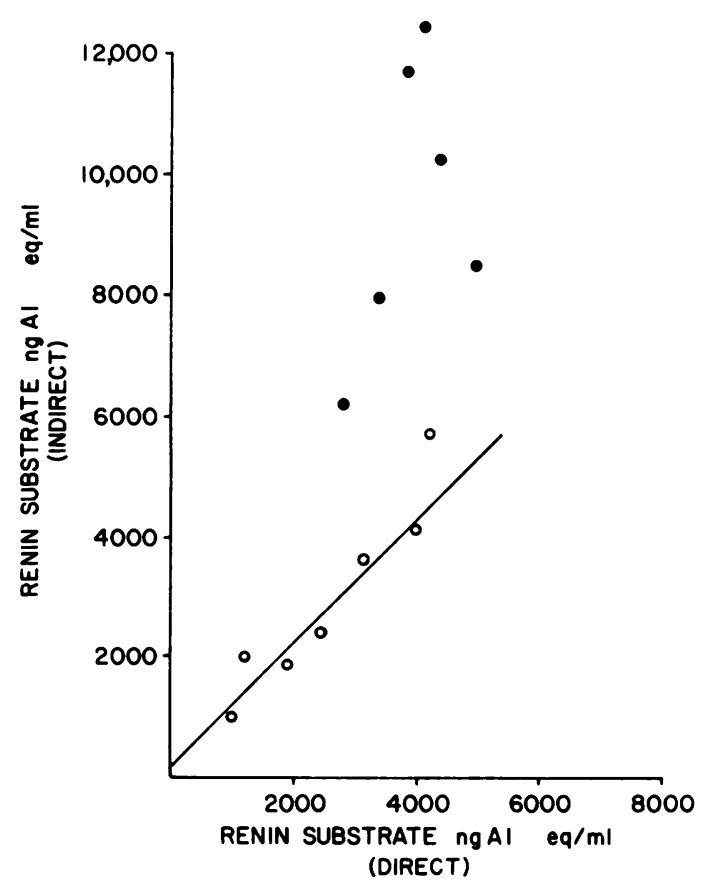

FIGURE 3 A comparison of direct and indirect assay for renin substrate in plasma of women on oral contraceptives. (O). Women demonstrating multiple forms of renin substrate on polyacrylamide gel electrophoresis at $\mathrm{pH} 8.4$; (O) women on oral contraceptives with no evidence of multiple forms of substrate by polyacrylamide gel electrophoresis at pH 8.4. from several types of hypertensive subjects (Fig. 5). In plasma from uremic hypertensive subjects, five of nine showed a discrepancy between the assay methods; again more substrate was evident by the indirect method. On polyacrylamide gel electrophoresis, at $\mathrm{pH}$ 8.4, multiple forms, similar to those seen in the plasma of women on oral contraceptives, were evident. Similar results were also seen in 3 of 12 patients with advanced essential hypertension. In contrast, no discrepancy between the assay methods (Fig. 5) and a

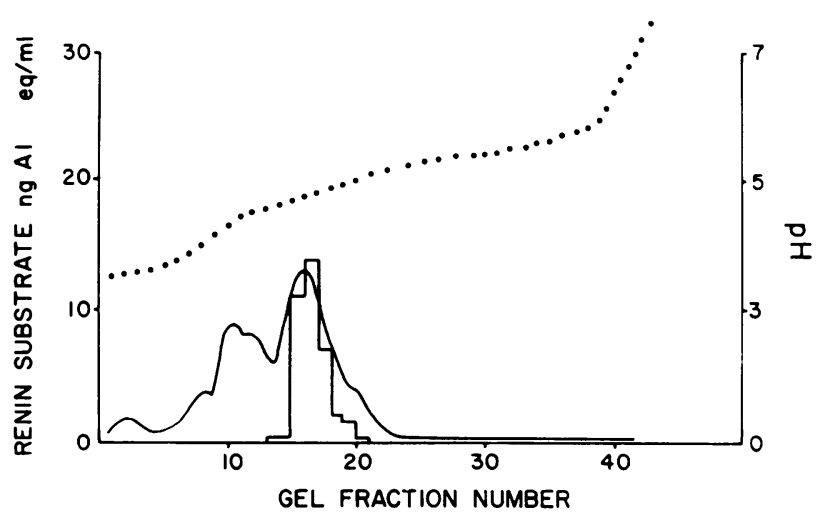

Figure 4 Plasma of patient with Cushing's syndrome on isoelectric focusing in a polyacrylamide gel. Bars indicate substrate determined by direct and continuous line indirect assay; dotted line indicates $\mathrm{pH}$ profile. 


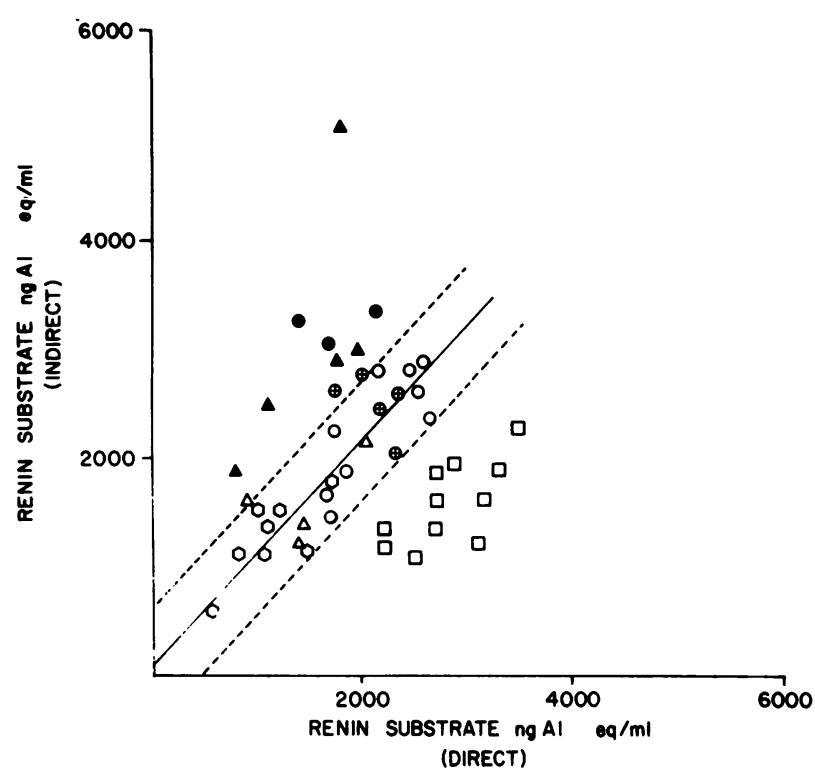

FIGURE 5 A comparison of direct and indirect assay for renin substrate. Open symbols, except for $\square$, indicate a 1:1 correlation between methods, corresponding closed symbols are those of patients in the same category showing multiple forms of renin substrate by polyacrylamide gel electrophoresis at $\mathrm{pH}$ 8.4. Solid line indicates least square linear repression line from normotensive subjects, dashed line indicates 1 SD. Advanced essential hypertension $(0,0)$, uremia associated with hypertension $(\triangle, \Delta)$, malignant hypertension $(\oplus)$, cirrhosis of the liver $(O)$ and renovascular hypertension ( $\square)$.

normal gel electrophoresis pattern ( $\mathrm{pH} 8.4)$ was observed in patients with malignant hypertension ( $n$ $=5$ ). In eight patients with suppressed renin substrate levels associated with cirrhosis of the liver (Fig. 5), no abnormalities were evident.

Patients with renovascular hypertension were the only subjects to show a different type of discrepancy between assay methods; the direct assay gave higher substrate concentrations than determinations using angiotensin I generation $(3,320 \pm 480$ [SD] vs. 1,840 \pm 455 [SD] $\mathrm{ng}$ angiotensin $\mathrm{I} \mathrm{eq} / \mathrm{ml}$ ), Fig. 5. Polyacrylamide gel electrophoresis of these plasmas at $\mathrm{pH}$ 8.4 revealed only the normal renin substrate peak, $\mathrm{Rf}$ 0.65 but lacked a 1:1 correspondence. Upon electrophoresis at $\mathrm{pH} 4.3$, however, the direct radioimmunoassay indicated a large quantity of a protein which cross-reacted with the antiserum but did not generate angiotensin I. (Fig. 2D). The discrepancy observed between direct and indirect assay methods for renin substrate in plasma of patients with renovascular hypertension may, therefore, be attributed to high in vivo concentrations of this immunoreactive protein.

To investigate whether stimulated plasma renin levels comparable to those of patients with renovascular hypertension (PRA $>50 \mathrm{ng}$ angiotensin $\mathrm{I} / \mathrm{ml}$ per $h$ ), could lead to elevated levels of this immunoreactive protein, plasma samples from patients with essential hypertension were compared before and after stimulation of plasma renin by spironolactone. In eight patients, the mean PRA increased from $7.4 \pm 2.5$ (SD) to $58.7 \pm 7.1$ (SD) after spironolactone treatment. No statistically significant changes in renin substrate levels were evident and, despite the large increase in PRA, a 1:1 correspondence between the two assay methods was maintained. These results indicate that the level of this immunoreactive protein remains low in these subjects, despite the high levels of circulating renin.

Partial characterization of multiple forms of renin substrate. To obtain an estimate of the rates of angiotensin I generation with the various forms of renin substrate, polyacrylamide gel fractions were pooled and equal quantities of each substrate form were incubated for $20 \mathrm{~min}$ at $37^{\circ} \mathrm{C}$ in the presence of homologous renal renin. In this 20 -min time period, $15.6 \pm 4.7 \%$ of the major normal substrate (I) was consumed as compared to $3.6 \pm 1.7 \%$ for II $(\mathrm{Rf}=0.35)$ and $34.6 \pm 3.2 \%$ for III ( $R f=0.16)$, indicating an accelerated rate of angiotensin production from form III in comparison with the normal renin substrate, form I. The effects on the renin reaction of the immunoreactive protein from the renovascular hypertensive subjects were also studied. This protein fraction isolated from several electrophoresis runs at $\mathrm{pH} 4.3$ was pooled and increasing quantities were added to normal plasma. The inhibition of PRA is shown in Fig. 6; its influence on the rate of the renin reaction is also shown in Fig. 6. The data indicate that this protein acts as a competitive inhibitor of the renin reaction.

\section{DISCUSSION}

As shown in the present report, the lack of a $1: 1$ correspondence between the two methods for substrate quantitation, in certain subjects has led us to the discovery of multiple forms of renin substrate in human plasma. Multiple peaks of renin substrate activity have also been recently reported by other investigators $(10$, 11). In our studies, a lower estimate of plasma renin substrate concentration by direct radioimmunoassay compared to angiotensin I-generating capacity, was observed mainly in plasmas with elevated renin substrate levels. In these cases, plasma renin substrate was found to be heterogenous by either polyacrylamide gel electrophoresis or isoelectric focusing. Underestimation by the direct assay was most pronounced in those women on oral contraceptives with the highest plasma renin substrate concentrations. A corollary observation was made in women during the third trimester of pregnancy. Underestimation by the direct assay and multiple forms of renin substrate, evident 


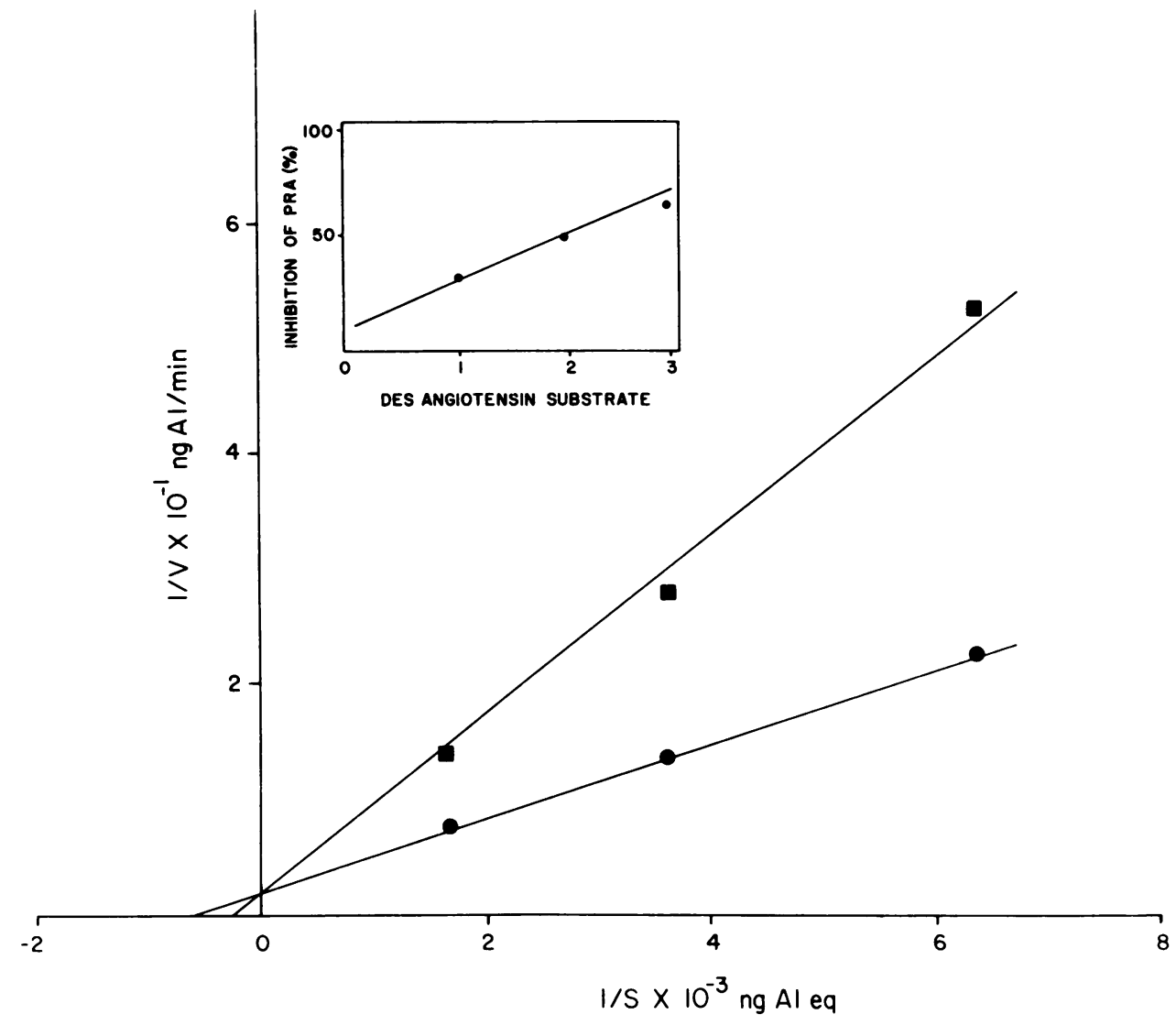

FIGURE 6 Rate of renin reaction in presence of the immunoreactive plasma protein (IP). Lineweaver-Burk plot of normal human plasma incubated at $\mathrm{pH} 7.4 \mathrm{with}$ added renin $(\Theta)$; same as above with added IP ( $\square$ ); insert shows percent inhibition of PRA by the addition of IP (arbitrary units).

during the last trimester, were no longer apparent postpartum, when renin substrate levels had returned to normal.

Heterogeneous renin substrate was also evident in certain pathological states. The uremic patients, on chronic hemodialysis having severe renal damage and elevated plasma renin substrate levels $(3,960 \pm 1,340$ [SD] vs. $2,110 \pm 790$ [SD] for normal subjects) also demonstrated multiple forms of renin substrate. An elevation of renin substrate levels in anephric or hemodialysis patients in the terminal stages of chronic renal failure has previously been reported (12). The present study also demonstrates that multiple forms of renin substrate are present not only in patients with high plasma estrogen levels, but also in patients receiving glucocorticoids.

In contrast to the above observations, overestimation of renin substrate levels by the direct assay due to a circulating immunoreactive renin substrate-like substance from which angiotensin I could not be generated by incubation with added homologous renin was found in statistically significant quantities, only in the plasma of patients with renovascular hypertension. We suggest that this protein is desangiotensin I renin substrate because the plasma concentration of this protein was $(a)$ shown to increase after incubation with renin, (b) cross-reacted completely with the antiserum to renin substrate and $(c)$ acted as a competitive inhibitor of the renin reaction. Although the physiological function of this protein is not known, it may be suggested that it acts as a feedback control mechanism for partial suppression of the renin reaction in plasma of patients with renovascular hypertension. This feedback mechanism would be of greatest significance in renovascular hypertension where substantial quantities of this protein were found to be circulating in plasma. Furthermore, in other types of hypertension, including essential hypertensive patients with high renin levels, acutely stimulated by spironolactone to plasma concentrations equivalent to those observed in renovascular hypertension, no significant quantities of this circulating immunoreactive 
renin inhibitor were evident. These results indicate, therefore, a possible adaptive alteration in the degradation process of this protein in renovascular hypertension.

We have also demonstrated in this study, new forms of renin substrate which are structurally different from the normal form $(\mathrm{Rf}=0.65)$ because they do not crossreact with the antibody specific for the normal form and differ in isoelectric points and electrophoretic mobilities. Furthermore, the new forms of renin substrate have altered kinetic parameters in the presence of added homologous renin.

In conclusion, the data support the postulate that stimulation of plasma renin substrate by either estrogens, glucocorticoids, or unknown factors such as observed in uremia may lead to the production of structurally new forms of renin substrate. Although the normal form of substrate is the major renin substrate form in all plasmas, the new forms of substrate may have a significant effect on the overall renin reaction in plasma because form III $(\mathrm{Rf}=0.16)$ appears to lead to an accelerated rate of angiotensin production and form II $(\mathrm{Rf}=0.35)$ decreases this rate. These new substrate forms may, therefore, possibly account for the altered kinetics of the renin reaction observed in some hypertensive states $(13,14)$.

\section{ACKNOWLEDGMENTS}

The authors would like to thank Dennis Phillips and Karen Dally for technical assistance.

This study was supported by Veterans Administration grant 7697 and National Institutes of Health grant 15039.

\section{REFERENCES}

1. Eggena, P., C. L. Chu, J. D. Barrett, and M. P. Sambhi. 1976. Purification and partial characterization of human angiotensinogen. Biochim. Biophys. Acta. 427: 208-217.

2. Eggena, P., J. D. Barrett, H. Hidaka, C. L. Chu, C. Thananopavern, M. S. Golub, and M. P. Sambhi. 1977.
A direct radioimmunoassay for human renin substrate and identification of multiple substrate types in plasma. Circ. Res. 41(Suppl. II): 34-37.

3. Barrett, J. D., P. Eggena, and M. P. Sambhi. 1976. Influence of angiotensinase inhibitors on the enzymatic activity of renin. Biochem. Med. 16: 157-168.

4. Haas, E., H. Goldblatt, E. C. Gipson, and L. Lewis. 1966. Isolation, purification and assay of human renin free of angiotensinase. Circ. Res. 19: 739-749.

5. Eggena, P., J. D. Barrett, M. P. Sambhi, and C. E. Wiedeman. 1974. The validity of comparing the measurements of angiotensin I generation in human plasma by radioimmunoassay and bioassay. J. Clin. Endocrinol. Metab. 39: 865-870.

6. Williams, C. A. 1968. Analytical zone electrophoresis. In Methods in Immunology and Immunochemistry. C. A. Williams and M. W. Chase, editors. Academic Press, Inc., New York. 19-56.

7. Lim, R., and E. Tadayyou. 1970. Fractionation of the insoluble brain proteins with acrylamide electrophoresis. Anal. Biochem. 34: 9-15.

8. Weinberger, M. H., R. D. Collins, A. J. Dowdy, G. W. Nokes, and J. A. Luetscher. 1969. Hypertension induced by oral contraceptives containing estrogen and gestagen. Ann. Intern. Med. 71: 891-902.

9. Cain, M. D., W. A. Walters, and K. J. Catt. 1971. Effects of oral contraceptive therapy on the renin-angiotensin system. J. Clin. Endocrinol. Metab. 33: 671-676.

10. Gordon, D. B., I. N. Sachin, and V. N. Dodd. 1976. Heterogeneity of renin substrate in human plasma: Effects of pregnancy and oral contraceptives. Proc. Soc. Exp. Biol. Med. 153: 314-318.

11. Printz, M. P., J. M. Printz, J. A. Lewicki, and W. J. Welch. 1977. Resolution of multiple forms of human angiotensinogen. Circ. Res. 41(Suppl. II): 37-43.

12. Nasjletti, A., L. A. Lewis, and G. M. C. Masson. 1971. Electrophoretic studies on renin substrate from various species. Am. J. Physiol. 220: 804-807.

13. Sambhi, M. P., and C. E. Wiedeman. 1972. Renin activation in venous plasma from the involved kidney in the patient with renal hypertension. J. Clin. Invest. 51: 22-30.

14. Sambhi, M. P., P. Eggena, J. D. Barrett, M. Tuck, C. E. Wiedeman, and C. Thananopavern. 1975. A circulating renin activator in essential hypertension. Circ. Res. 36, 37 (Suppl. I): 28-37. 